\title{
Cognitive representation of the comprehension test of safety warnings using multivariate methods
}

\author{
Cardoso-Junior, M.M. ${ }^{\mathrm{a},{ }^{*}}$ and Scarpel, R.A. ${ }^{\mathrm{b}}$ \\ a Office of Occupational Safety, Technological Institute of Aeronautics, Praça Marechal Eduardo Gomes, 50 - \\ Vila das Acácias, CEP 12.228-900 - São José dos Campos - SP - Brasil \\ ${ }^{\mathrm{b}}$ Department of Industrial Organization, Technological Institute of Aeronautics, Praça Marechal Eduardo Gomes, \\ 50 - Vila das Acácias, CEP 12.228-900 - São José dos Campos - SP - Brasil
}

\begin{abstract}
This paper presents a multivariate method to assess the comprehension of safety signs. The proposed method uses the MDS and Procrustes, to obtain a consensus perceptual map. The results show that the map allowed discriminating signs with high levels of comprehension from others. The safety signs have no statistical difference as depicted by Bootstrap Analysis.
\end{abstract}

Keywords: Cognitive, Multidimensional Scaling, Procrustes, Safety Warnings.

\section{Introduction}

The effectiveness of a safety warning involves a complex set of factors. A first approach is the evaluation or testing the design. Evaluation is the activity used to determine the degree to which the warning fulfills the objectives [11]. Evaluations may use subjective or objective measures, the first being the most used and usually the target group is asked to test the effectiveness of warnings in various dimensions with the use of Likert scales. Objective measures assess behavior (change) users against the warnings.

The expected contribution is to facilitate the work of comprehension test of warnings and the subsequent representation of same in a two-dimensional space, allowing to infer about possible biases in perception of the judge and variability of a warning.

\section{Multivariate Methods}

The method proposed in this study has three phases, analysis of interrater reliability judges, dimension reduction and adjustment of the various settings.

In the first phase the responses from the panelists are tested by Rv coefficient [1]. The goal is to detect possible outliers in the group of judges, which can compromise the final result.

In the second phase the data of the judges are subjected to multidimensional scaling - MDS. The MDS is a method that is based on the proximity of objects used to produce a spatial representation of them [6]. MDS is a dimension reduction technique, since its goal is to find a set of points in low dimension that reflects the configuration data in high dimension.

In third phase, settings obtained in phase 2 are then subjected to Generalized Procrustes Analysis - GPA, which aims to determine to what extent the different configurations obtained from each judge are in agreement [9] .

\footnotetext{
*Corresponding author. E-mail: moacyr@ita.br.
} 
This problem according to the author can be described as the measure of similarity between the $\mathrm{m}$ configurations, or interrater reliability judge.

After the GPA, we obtain the consensus configuration. All settings are aligned to this consensus, that is, each individual judgment undergoes a process of translation, scaling and rotation so that the difference between consensus and all other are minimal. The mismatch of configurations, regarding the dimensions, objects, and judges can also be evaluated with the aid of the variance of Procrustes, called PANOVA [4,9].

Finally the aligned configurations of GPA were submitted to bootstrap in order to obtain confidence regions.

To test the proposed method a set of safety warnings were selected, so that the four basic types would had to be included, Warnings, Prohibition, Mandatory and Information, as described in [2]. A total of 12 signs were distributed in the four basic types described above, considering the examples of [2]. Some safety signs presented a comprehension test of $85 \%$ or above and others $50 \%$ or less.

To construct the perceptual map, it was used the questionnaire suggested by [3], termed "cognitive assessment questionnaire". This questionnaire considers five attributes for cognitive assessment, which are: familiarity, concreteness, simplicity, meaning and semantic proximity.

The MDS solution was achieved using SMACOF package [5]. The GPA was determined by FactoMineR package [7] and the Bootstrap using Bootstrap package [10]. All of them implemented in $\mathrm{R}$ - CRAN Version 2.9.2 [8].

\section{Results}

Analyzing the ratio Rv can be said that there was no outlier in the group studied, and hence can consider all the judges in the analysis.

The questionnaires of the 17 panelists were then submitted to MDS for dimension reduction and then to obtain the GPA consensus configuration. Fig 1 shows the perceptual map obtained.

One can check that the perceptual map can discriminate Warnings for comprehension. The right side contains warnings with values above $85 \%$ of comprehension and the left one value less than $50 \%$ of comprehension.
Figure 1

Cognitive representation of the safety signs

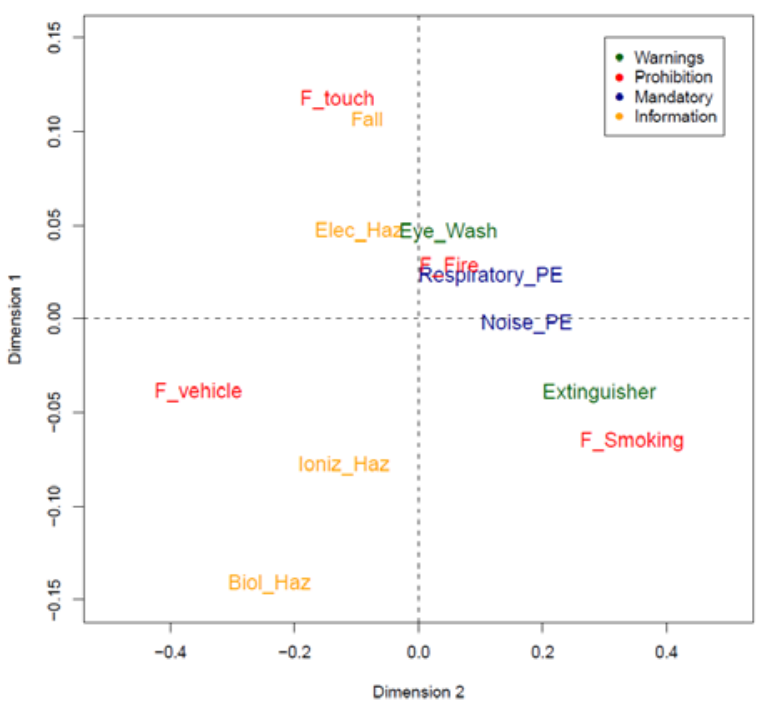

Although the analysis of the confidence regions points out in other direction, there are no statistical differences among safety signs comprehension. It can be seen in Fig. 2, that confidence regions are overlapped. The confidence regions in this case are only $5 \%$. For higher confidence levels like $75 \%, 90 \%$ and $95 \%$ there are no separation of the safety signs, which means they are all the same in a group interpretation.

Figure 2

Confidence regions of safety signs comprehension

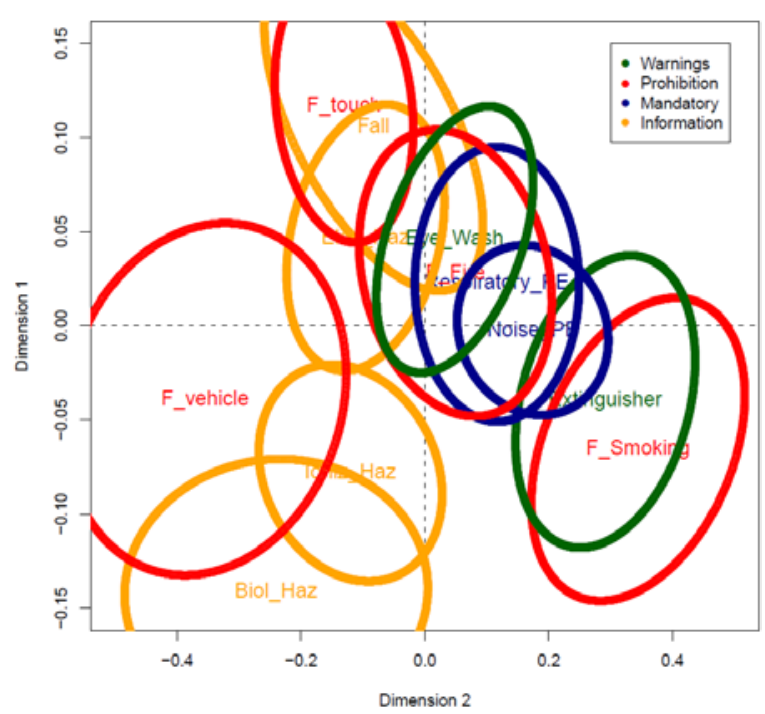




\section{Final remarks}

The perceptual map could portray the cognitive structure of respondents, since the second dimension separates into two groups Warnings: Warnings with high comprehension $(>85 \%)$ and the low ones $(50 \%)$.

However, it was not possible to state that one safety sign is statistically different from others.

Future works include the attempt to cluster the judges in order to produce more than one perceptual map to represent the cognitive structure of the group.

\section{References}

[1] Abdi, H.; Dunlop, J. P.; Williams, L. J. (2009) How to compute reliability estimates and display confidence and tolerance intervals for pattern classifiers using Bootstrap and 3-way multidimensional scaling (DISTATIS). Neuroimage.

[2] ANSI Z 535-3, I. A. N. S. (2007) Criteria for Safety Symbols. New York: ANSI.

[3] Chan, A. H. S.; Ng, A. W. Y. (2010) Investigation of guessability if industrial safety signs: Effects of prospective-user factors and cognitive sign features. International Journal of Industrial Ergonomics, 40. 689-697.

[4] Dijksterhuis, G. B.; Gower, J. G. (2010) The interpretations of generalized Procrustes analysis and allied methods. Urtecht.

[5] De Leeuw, J.; Mair, P. (2009). Multidimensional Scaling Using Majorization: SMACOF in R. Journal of Statistical Software, 31(3), 1-30. URL http://www.jstatsoft.org/v31/i03/

[6] Hardle, W.; Simar, L. (2007) Applied Multivariate Statistical Analysis. 2 Ed. ed. Berlin: Springer.

[7] Husson, F. e Josse, J., Le, S. Mazet, J., 2009. FactoMineR: Factor Analysis and Data Mining with R. R package version 1.12. Source: $<$ http://CRAN.R-project.org/package $=$ FactoMineR>. Acess: Jan.15 2009.

[8] R Development Team (2009), R: A language and environmen for statistical computing. Vienna: R. Foundation for Statistical Computing.

[9] Rodrigue, N. A (1999) Comparison of the Performance of Generalized Procrustes Analysis and the Intraclass Coefficient of Correlation to Estimate Interrater Reliability. Montreal: McGill University.

[10]S original, from StatLib and by Rob Tibshirani. R port by Friedrich Leisch. (2007). bootstrap: Functions for the Book "An Introduction to the Bootstrap". R package version 1.0-22.

[11] Wogalter, M. S.; Conzola, V. C.; Smith-Jackson, T. L. (2002) Research-based guidelines for warning design and evaluation. Applied Ergonomics, 33. 219-230. 